\title{
LOOKING INTO PULSAR MAGNETOSPHERES
}

\author{
MAXIM LYUTIKOV \\ Canadian Institute for Theoretical Astrophysics, 60 St. George Street, Toronto, Canada
}

\begin{abstract}
Diffractive and refractive magnetospheric scintillations may allow direct probing of the plasma inside the pulsar light cylinder. The unusual electrodynamics of the strongly magnetized electron-positron plasma allows separation of the magnetospheric and interstellar scattering. The most distinctive feature of the magnetospheric scintillations is their independence of frequency. Diffractive scattering due to small scale inhomogeneities produces a scattering angle that may be as large as 0.1 radians, and a typical decorrelation time of $10^{-8}$ seconds. Refractive scattering due to large scale inhomogeneities is also possible, with a typical angle of $10^{-3}$ radians and a correlation time of the order of $10^{-4}$ seconds. Some of the magnetospheric propagation effects may have already been observed.
\end{abstract}

A number of observational results may possibly be attributed to scattering processes inside pulsar magnetospheres. The most convincing are the results of Sallmen et al. (1999) in which the frequency independent spread and the multiplicity of the Crab giant pulses with large variations in the pulse broadening times. Other observations that can possibly be attributed to magnetospheric propagating effects include an unusual scaling of broadening times for giant pulses (Hankins and Moffett, 1998), 'ghost' images of the Crab pulse and interpulse (Smith and Lyne, 1999; Backer, 1999), enhanced intensity fluctuations at very high frequencies (Kramer $e t$ al., 1997), enhanced scattering of nearby pulsars (Gupta et al., 1994; Rickett et al., 1999), the comparatively large size of the Vela pulsar's radio emission region (Gwinn et al., 1997), the absence of microstructure with very short time scales (Popov, 1999), and frequency independent scintillations with a bandwidth $4 \mathrm{MHz}$ (Gwinn et al., 1999).

Strong magnetic fields present in pulsar magnetospheres and the unusual electrodynamics of the one-dimensional electron-positron plasma both change the familiar effects of scattering and refraction in a plasma. The unusual features of scattering in such a plasma may allow its separation from the interstellar scattering and serve as a tool to probe the structure of the magnetosphere itself. Typically, the frequency of the observed radio waves is much less that the cyclotron frequency $\omega \ll \omega_{B}$. For such frequencies the refractive index of a strongly magnetized pair plasma for the escaping electromagnetic modes is approximately

$$
\delta \equiv n^{2}-1 \approx \frac{\gamma_{p} \omega_{p}^{2}}{\omega_{B}^{2}}=3 \times 10^{-4}\left(\frac{P}{0.1 \mathrm{sec}}\right)^{2} .
$$

Thus, the large Lorentz factor of the moving plasma effectively enhances the waveplasma interaction on the open field lines. The parameter $\delta$ is the key to the scatter- 
ing and diffraction effects in the pulsar magnetosphere. An important fact is that $\delta$ does not strongly depend on our assumptions about the density and the streaming Lorentz factors of the plasma.

The parameter $\delta$, which determines refractive properties of the medium, is negligible deep inside the pulsar magnetosphere, but increases with distance from the neutron star as $\propto r^{3}$. Thus, the strongest nonresonant wave-plasma interactions occur in the outer regions of pulsar magnetospheres (near the light cylinder). This allows a considerable simplification when considering scattering and diffraction effects since one can adopt a 'thin screen' approximation. We assume that emission is generated deep in the pulsar magnetosphere and then scattered in a thin screen located near the light cylinder with a typical thickness $D \approx 0.1 R_{L C}$.

Two types of inhomogeneities should be present inside the pulsar magnetosphere: small scale inhomogeneities with typical sizes comparable to tens of a skin depth $\sim c / \omega_{p}$, which arise from the plasma turbulence, and large scale inhomogeneities with typical sizes comparable to the light cylinder radius which arise from temporal and spatial modulation of the outflowing pair plasma. The two types of inhomogeneities will produce qualitatively different effects: small scale inhomogeneities will produce diffractive scattering, while large scale inhomogeneities will produce refractive scattering. As usual, the scale that defines 'small' and 'large' is the Fresnel scale which in this case is equal to $r_{f}=\sqrt{R_{L C} \lambda} \sim 10^{5} \mathrm{~cm}$.

Assuming that scattering inside the pulsar magnetosphere is strong, the typical scattering angle can be estimated as

$$
\theta_{\text {scat }} \approx \frac{\Delta \phi}{2 \pi} \frac{\lambda}{a}=\left(\frac{D}{a}\right)^{1 / 2} \delta \Delta n_{e}
$$

where $D \sim 0.1 R_{L C}$ is the thickness of the scattering screen, $a$ is a typical size of irregularities and $\Delta n_{e}$ is the relative amplitude of density fluctuations.

A necessary requirement to allow for the separation of scintillations into diffractive and refractive branches is that the scattering should be strong, so that the total phase shift of the wave is much larger than $\pi$. For a medium with given size inhomogeneities the scattering is strong for

$$
\lambda<\lambda_{\max }=\sqrt{a D} \delta \approx\left\{\begin{array}{l}
30 \mathrm{~cm} \text { for } a=a_{\min } \\
10^{5} \mathrm{~cm} \text { for } a=a_{\max }
\end{array}\right.
$$

This is a limitation of the wavelength from above: scattering is stronger for shorter wavelengths. This is in sharp contrast to the scattering in the interstellar medium, where the strength of the scattering increases at low frequencies.

For the typical wavelength of observations $\lambda=30 \mathrm{~cm}$, both refractive and diffractive scattering occurs in a strong scattering regime with the total phase change:

$$
\Delta \phi \approx \begin{cases}1 & \text { diffractive } \\ 100 & \text { refractive }\end{cases}
$$

Diffractive scattering becomes weak at longer wavelengths. 
Assuming that there are strong density fluctuations, $\Delta n_{e} \sim 1$, present on smallest scales $a \sim a_{\text {min }}$ the diffractive scattering angle becomes $\theta_{D} \approx 0.1 \mathrm{rad}$. This implies that large scattering is possible in the outer regions of pulsar magnetospheres. If this extreme scattering case was realized, the observed profiles are then would be a convolution of the 'initial' window function (determined by the emission conditions at lower radii) with diffractive scattering. Since this is not what is observed, we should conclude that strong density fluctuations are not present at very small scales.

On the other hand, strong refractive density fluctuations are almost definitely present inside the pulsar magnetosphere. The typical refractive scattering angle is

$$
\theta_{R} \approx \delta=4 \times 10^{-3}
$$

Refractive effects will induce 'jitter' in the arrival times of the pulses and a temporal correlation in the intensities with a typical scale $\tau_{R}=\theta_{R} P=4 \times 10^{-4} \mathrm{sec}$. Both of these effects will be independent of frequency, and will increase with the period of the pulsar.

Scattering may also affect the polarization structure of emission. If two regions in the magnetosphere emit different polarization, scattering may mix the radiation decreasing the degree of polarization. The amount of mixing will be different in the weak scattering regime at frequencies $\leq 1 \mathrm{GHz}$ and strong scattering regime at $\geq 1 \mathrm{GHz}$. This effect may be responsible for the high frequency depolarization observed in some pulsars (I thank Barney Rickett for pointing out this possibility). Also note that real change of the polarization vector of the radiation is impossible in the outer regions of the magnetosphere, beyond the limiting polarization surface.

Another possible effect of propagation inside the pulsar magnetosphere includes reflection from the boundary between the closed and open field lines. The plasma density on the closed field lines may approach a value at which the thermal energy density of plasma reaches the magnetic field energy density. For mildly relativistic plasma with a thermal velocity of the order of the velocity of light the maximum plasma density, $n \sim B^{2} /\left(8 \pi m c^{2}\right)$.

Experiments to detect the effects of wave propagation inside the pulsar magnetosphere should use high frequencies and concentrate on nearby pulsars of low dispersion measure. Possible experiments will include a search for nondispersive effects such as a time delay (as large as tens of microseconds) in the pulse arrival, a diffractive decorrelation bandwidth of the order of $10 \mathrm{MHz}$, and microstructure periodicities (of the order of tens of microseconds) due to refractive scattering. Nearby strong pulsars, like PSR 0950, are the best candidates to search for magnetospheric effects.

The predicted characteristics of the scattering inside the pulsar magnetosphere are

diffractive scattering angle

$10^{-1}$

diffractive scattering time

$10^{-4} \mathrm{sec}$ 
diffractive decorrelation time refractive scattering angle refractive decorrelation time arrival time variations

$$
\begin{aligned}
& 10^{-8} \mathrm{sec} \\
& 10^{-3} \\
& 10^{-4} \mathrm{sec} \\
& 10^{-4} \mathrm{sec}
\end{aligned}
$$

\section{References}

Backer, D.C.: 2000, in: M. Kramer, N. Wex and N. Wielebinski (eds.), Pulsar Astronomy - 2000 and Beyond, IAU Colloquium 177,493.

Gwinn, C.R., et al.: 1997, Astrophys. J. 483, L53.

Gwinn, Hirano and Britton: 1999, personal communication.

Gupta, Y., Rickett, B.J. and Lyne, A.G.: 1994, Mon. Not. R. Astron. Soc. 269, 1035.

Popov, M.V., et al.: 2000, in: M. Kramer, N. Wex and N. Wielebinski (eds.), Pulsar Astronomy 2000 and Beyond, IAU Colloquium 177, 179.

Rickett, B.J., Coles, W.A. and Markkanen, J.: 1999, 2000, ApJ 533, 304.

Sallmen et al.: 1999, Astrophys. J. 517, 460.

Smirnova et al.: 1996, Astrophys. J. 462, 289.

Smith, F.G. and Lyne, A.G.: 2000, in: M. Kramer, N. Wex and N. Wielebinski (eds.), Pulsar Astronomy - 2000 and Beyond, IAU Colloquium 177, 499. 